\title{
Aplicação do Cálculo Exterior Discreto para Simulação Numérica de Sistemas de GPR
}

\author{
A. S. Moura ${ }^{1}$, W. G. Facco ${ }^{2}$ \\ ${ }^{1}$ Departamento de Economia, Universidade Federal de Juiz de Fora,Brazil \\ ${ }^{2}$ Coordenadoria de Engenharia Mecânica, Instituto Federal do Espírito Santo, Brasil
}

Palavras-chave: Formas Diferenciais, Equações de Maxwell, Propagação de onda, GPR.

Resumo: Apresenta-se neste trabalho o uso das formas diferenciais através da teoria do Cálculo Exterior Discreto para a simulação de uma classe de problemas de propagação de ondas eletromagnéticas, a saber os sistemas de radar de penetração de solo. Equações de Maxwell modificadas serão utilizadas na região de absorção do dominio computacional, esta abordagem é denominada CFS-PML (complex frequency shifted-perfectly matched layer). A discretização proposta neste artigo garante tanto a simplificação do algoritmo, como uma formulação mais elegante das expressões. As Equações de Maxwell são resolvidas no domínio do tempo em termos de campos elétrico e magnético, com a utilização de um o esquema do tipo leap-frog acoplado com uma convolução recursiva dentro da região PML de absorção. Dois exemplos serão apresentados, onde se trata a modelagem de cenários típicos de sistemas de radar de penetração de solo, que incluem meios com perdas.

\section{Introdução}

Para que se tenha um modelamento eficiente dos fenômenos eletromagnéticos envolvendo aplicações de Radar de Penetração de Solo ( Ground Penetrating Radar- GPR) ${ }^{1}$, é necessário a utilização de técnicas numéricas que resolvam problemas físicos com um grau elevado de dificuldade. Logo, a motivação principal deste trabalho está na aplicação de um método numérico para a solução de problemas que envolvam o uso dos georadares.

Neste artigo utiliza-se a teoria das formas diferenciais que fornece uma estrutura matemática para a teoria de campos eletromagnéticos [1]. O uso das formas diferenciais está baseado na representação das equações de Maxwell através da construção de dois sistemas matriciais em termos da intensidade do campo elétrico $\vec{E}$ (representado como a formulação primal) e a intensidade do campo magnético $\vec{H}$ (denotada como a formulação dual), respectivamente. A conexão entre a formulação primal e dual é estabelecida através do operador estrela de Hodge [2].

\section{Equações de Maxwell Modificadas}

Em um domínio bidimensional, as equações de Maxwell em termos do campo elétrico $\mathcal{E}$ e da densidade de fluxo magnético $\mathcal{B}$ dentro da região de absorção [3], serão descritas por:

$$
\begin{gathered}
\frac{d \mathcal{B}}{d t}=-\mathrm{d}(\overline{\bar{\wedge}}(t) * \mathcal{E}) \\
\mathrm{d}\left(\overline{\bar{\wedge}}(t) *\left(\star_{\nu} \mathcal{B}\right)\right)=\star_{\epsilon} \frac{d \mathcal{E}}{d t}+\star_{\sigma} \mathcal{E}
\end{gathered}
$$

onde $*$ indica a convolução no tempo, d a derivada exterior e $\star$ o operador estrela de Hodge.

\footnotetext{
${ }^{1}$ Será usado em todo o texto a sigla em inglês para Radar de Penetração de Solo.
} 
As coordenadas de $\overline{\bar{\lambda}}(t)$ são definidos por:

$$
\frac{1}{S_{q}(t)}=\frac{\delta(t)}{k_{p}}-\frac{\sigma_{p}}{\epsilon_{0} k_{p}^{2}} e^{-\left(\frac{\sigma_{p}+\alpha k_{p}}{\epsilon_{0} k_{p}}\right) t} u(t)
$$

Fora da região de absorção assumi-se $\overline{\bar{\wedge}}(t)=1$ para se ter as equações clássicas de Maxwell. $\delta(t)$ é o delta de Dirac e $u(t)$ é a função Heaviside, os parâmetros PML na Eq.(3) são determinados por:

$$
k=1+\left(k_{\max }-1\right)\left(\frac{\rho}{d}\right)^{m}
$$

onde $k_{\max }$ é o máximo valor de $k$ na fronteira exterior. A condutividade $\sigma$ é descrita como sendo

$$
\sigma=\sigma_{\max }\left(\frac{\rho}{d}\right)^{m}
$$

onde $\sigma_{\max }$ é a condutividade máxima.

$$
\sigma_{\max }=-\frac{m+1}{2 \eta} d \ln (R)
$$

e $\eta$ é a impedância intrínseca do meio, e $R$ é o coeficiente teórico de reflexão para uma incidência normal e $m$ é a ordem do perfil utilizado e $\alpha$ é uma constante não dimensionada.

\section{Equações Modificadas de Maxwell Semi-discretas}

Considere a aproximação dos campos restritos aos elementos do domínio bidimensional $\Omega$ dada por:

$$
\begin{aligned}
\mathcal{E} & =\sum_{i=1}^{3} e_{i} \omega_{i}^{1} \\
\mathcal{B} & =\sum_{m=1}^{1} b_{m} \omega_{m}^{2}
\end{aligned}
$$

onde $\omega_{i}^{p}, p=1,2$, é a $p$-forma de Whitney [4], associada com o i-ésimo $p$-simplexo. Os coeficientes $e_{i}$ e $b_{m}$ são a tensão elétrica ao longo da $i$-ésima aresta e o fluxo magnético através da $m$-ésima face $f_{m}$, respectivamente.

Discretizando-se o domínio $\Omega$ por uma malha simplicial [5], e utilizando as equações (7) e (8) em (1) e expandido-as para uma soma de componentes cartesianos, obtêm-se para cada elemento $f_{m}$ a seguinte equação:

$$
\sum_{i=1}^{3}\left(\sum_{q=\{x, y\}} \mathrm{d}\left(\omega_{i q}^{1}\right)\left(\frac{1}{S_{q}(t)} * e_{i}\right)\right)=-\omega_{m}^{2} \frac{d b_{m}}{d t}
$$

onde $\omega_{i q}^{1}$ são as componentes da 1-forma de Whitney para $q=\{x, y\}$ e $m$ é um número inteiro que identifica o triângulo na malha.

Utilizando-se as propriedades do produto exterior [3], a equação (9) pode ser escrita da seguinte forma:

$$
\sum_{i=1}^{3}\left(\frac{1}{2} \mathrm{~d}\left(\omega_{i}^{1}\right) \sum_{q=\{x, y\}}\left(\frac{1}{S_{q}(t)} * e_{i}\right)\right)=-\omega_{m}^{2} \frac{d b_{m}}{d t} .
$$

Após a aplicação do Teorema Generalizado de Stokes [6] na variedade triangular,T, a forma semi-discreta de (10) é obtida,

$$
\frac{1}{2}[( \pm) 1( \pm) 1( \pm) 1] .\left[\sum_{q=\{x, y\}}\left(\frac{1}{S_{q}(t)} *\left[\begin{array}{l}
e_{1} \\
e_{2} \\
e_{3}
\end{array}\right]\right)\right]=\partial_{t} b_{m} .
$$


Considerando a soma sobre todos os triângulos da malha na região PML, o sistema semidiscreto global de equações diferenciais ordinárias é

$$
\frac{d \mathbf{b}}{d t}=-\frac{1}{2} \mathbf{C} \sum_{q=\{x, y\}} \mathbf{e}_{q}^{*}
$$

onde $\mathbf{C}$ é uma matriz $N_{f} \times N_{e}$ que representa a derivada exterior discreta agindo sobre 1-formas discretas [7], e e $\mathbf{b}$ são vetores coluna de incógnitas e $\mathbf{e}_{q}^{*}$ é dado por:

$$
\mathbf{e}_{q}^{*}=\left[\frac{1}{S_{q}(t)} * e_{1}(t), \frac{1}{S_{q}(t)} * e_{2}(t), \ldots, \frac{1}{S_{q}(t)} * e_{N_{e}}(t)\right]
$$

Utilizando-se o método de Galerkin e as propriedades fundamentais dos espaços Whitney [6], a discretização da equação (2), para um triângulo será descrita por:

$$
\sum_{q=\{x, y\}}\left(\left(\frac{1}{S_{q}(t)} * b_{m}\right)\left[\begin{array}{c} 
\pm 1 \\
\pm 1 \\
\pm 1
\end{array}\right] \mathbf{M}_{2}(\nu)_{m m}\right)=\left[\mathbf{M}_{1}(\sigma)_{i j}\right] e_{i}+\left[\mathbf{M}_{1}(\epsilon)_{i j}\right] \frac{d e_{i}}{d t}
$$

onde $i, j=1,2,3$ e $\mathbf{M}_{1}(\epsilon), \mathbf{M}_{1}(\sigma)$ e $\mathbf{M}_{2}(\nu)$ são matrizes de Hodge [7], definidas por:

$$
\mathbf{M}_{p}(\alpha)=\int_{\Omega} \alpha w_{p}^{i} \cdot w_{p}^{j} d \Omega
$$

onde $\alpha$ denota um dos campos escalares $\epsilon, \nu, \sigma$ e $p$ o grau da forma diferencial. Através da assemblagem de todos os elementos da malha teremos a seguinte equação matricial

$$
\mathbf{M}_{1}(\epsilon) \frac{d \mathbf{e}}{d t}=\frac{1}{2} \mathbf{C}^{T} \mathbf{M}_{2}(\nu)\left[\sum_{q \in\{x, y\}}\left(\frac{1}{S_{q}} * \mathbf{b}\right)\right]-\mathbf{M}_{1}(\sigma) \mathbf{e} .
$$

onde $\mathbf{b}_{q}^{*}$, se expressa da seguinte forma:

$$
\mathbf{b}_{q}^{*}=\left[\frac{1}{S_{q}(t)} * b_{1}(t), \frac{1}{S_{q}(t)} * b_{2}(t), \ldots, \frac{1}{S_{q}(t)} * b_{N_{f}}(t)\right]
$$

Usando (3), cada componente dos vetores convoluídos em (13) e (17), pode ser escrita da seguinte maneira, respectivamente:

$$
\begin{aligned}
& \frac{1}{S_{q}(t)} * e_{i}(t)=\frac{1}{k_{q, i}} e_{i}(t)+\phi_{q, i}(t) \\
& \frac{1}{S_{q}(t)} * b_{j}(t)=\frac{1}{k_{q, i}} e_{i}(t)+\psi_{q, j}(t)
\end{aligned}
$$

onde

$$
\begin{gathered}
\xi_{q, i}(t)=-\frac{\sigma_{q, i}}{\epsilon_{0} k_{q, i}^{2}} e^{-\left(\frac{\sigma_{q, i}+\alpha k_{q}}{\epsilon_{0} k_{q, i}}\right) t} u(t) \\
\phi_{q, i}(t)=\xi_{q, i}(t) * e_{i}(t) \\
\psi_{q, j}(t)=\xi_{q, j}(t) * b_{j}(t) .
\end{gathered}
$$

A representação discreta da equação (20), pode ser dada por:

$$
\begin{aligned}
\xi_{q, i}[n] & =\int_{n \Delta t}^{(n+1) \Delta t} \xi_{q, i}(\tau) d \tau \\
& =g_{q, i} e^{-\left(\frac{\sigma_{q, i}+\alpha k_{q}}{\epsilon_{0} k_{q, i}}\right) n \Delta t}
\end{aligned}
$$


onde,

$$
\begin{gathered}
g_{q, i}=\left(\frac{\sigma_{q, i}}{k_{q, i}\left(\sigma_{q, i}+\alpha k_{q}\right)}\right)\left(h_{q, i}-1\right) \\
h_{q, i}=e^{-\left(\frac{\sigma_{q, i}+\alpha k_{q}}{\epsilon_{0} k_{q, i}}\right) \Delta t} .
\end{gathered}
$$

Por (23)-(25) é possível escrever $\xi_{q, i}[n+1]$ em função de $\xi_{q, i}[n]$,

$$
\xi_{q, i}[n+1]=\xi_{q, i}[n] g_{q, i} .
$$

Por (26) obtêm-se a forma discreta das convoluções em (21) e (22),

$$
\begin{aligned}
\phi_{q, i}[n+1] & =h_{q, i} e_{i}(n+1)+g_{q, i} \phi_{q, i}[n] \\
\psi_{q, j}[n+1] & =h_{q, j} b_{j}(n+1)+g_{q, j} \psi_{q, j}[n]
\end{aligned}
$$

Assim a discretização no tempo das equações em 12 e 16, com a aplicação de um esquema leap-frog para resolver o sistema é dada por

$$
\begin{gathered}
\mathbf{b}^{n+\frac{1}{2}}=\mathbf{b}^{n-\frac{1}{2}}-\frac{\Delta t}{2} \mathbf{C} \sum_{q}\left(\mathbf{K}_{q, e}^{n}+\mathbf{\Phi}_{q}^{n}\right) \\
\mathbf{N}_{1} \mathbf{e}^{n+1}=\mathbf{M}_{1}(\epsilon) \mathbf{e}^{n}+\frac{\Delta t}{2} \mathbf{C}^{T} \mathbf{M}_{2}(\nu) \sum_{q}\left(\mathbf{K}_{q, b}^{n+\frac{1}{2}}+\mathbf{\Psi}_{q}^{n+\frac{1}{2}}\right)
\end{gathered}
$$

onde

$$
\begin{gathered}
\mathbf{N}_{1}=\left(\mathbf{M}_{1}(\epsilon)+\Delta t \mathbf{M}_{1}(\sigma)\right) \\
\mathbf{\Phi}_{q}(t)=\left[\phi_{q, 1}(t), \phi_{q, 2}(t), \cdots, \phi_{q, N_{e}}(t)\right] \\
\mathbf{\Psi}_{q}(t)=\left[\psi_{q, 1}(t), \psi_{q, 2}(t), \cdots, \psi_{q, N_{f}}(t)\right] \\
\mathbf{K}_{q, e}(t)=\left[e_{1}(t) / k_{q, 1}, e_{2}(t) / k_{q, 2}, \cdots, e_{N_{e}}(t) / k_{q, N_{e}}\right] \\
\mathbf{K}_{q, b}(t)=\left[b_{1}(t) / k_{q, 1}, b_{2}(t) / k_{q, 2}, \cdots, b_{N_{f}}(t) / k_{q, N_{f}}\right]
\end{gathered}
$$

\section{Simulações Numéricas}

\subsection{Levantamento de superfícies baseados em reflexão - $2 \mathrm{~d}$}

Neste exemplo, será simulado um levantamento de superfícies baseado na reflexão, este sistema de GPR possui uma antena transmissora e receptora se encontram localizadas sobre a superfície da terra. Figura 1 apresenta a geometria computacional que é composta por três meios $\Omega_{1}, \Omega_{2}$ e $\Omega_{3}$ que representam respectivamente o ar, solo com permeabilidade relativa $\epsilon_{r}=9$, permissividade relativa $\mu_{r}=1$ e condutividade $\sigma=1 \mathrm{mS} / \mathrm{m}$ e outro solo com permeabilidade relativa $\epsilon_{r}=25$, permissividade relativa $\mu_{r}=1$ e condutividade $\sigma=5 \mathrm{mS} / \mathrm{m}$. Dentro das regiões $\Omega_{1}$ e $\Omega_{2}$ são inseridos dois objetos com paredes eletricamente perfeitas.

A excitação tem a forma de um pulso Blackman-Harris no ponto $(x, y)=(-1,2.5)$ com frequência dominante de $300 \mathrm{MHz}$. Os parâmetros da função CFS-PML são $m=3, R=$ $\exp (-9), k_{\max }=4$ e $\alpha=0.004$, a região PML possui uma espessura de $r=1,5 \mathrm{~cm}$, e é terminada por uma PEC.

Figura 2 mostra fotografias da componente $\mathcal{E}_{y}$ do campo elétrico em vários instantes de tempo. É observado a propagação da onda através do ar e sua entrada na região de PML, 


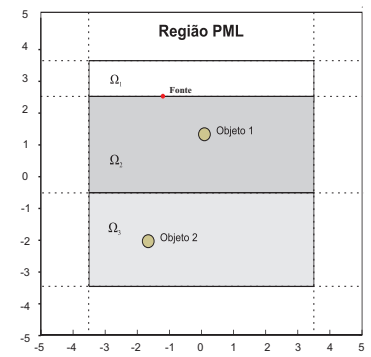

Figura 1: Modelo com propriedades elétricas.
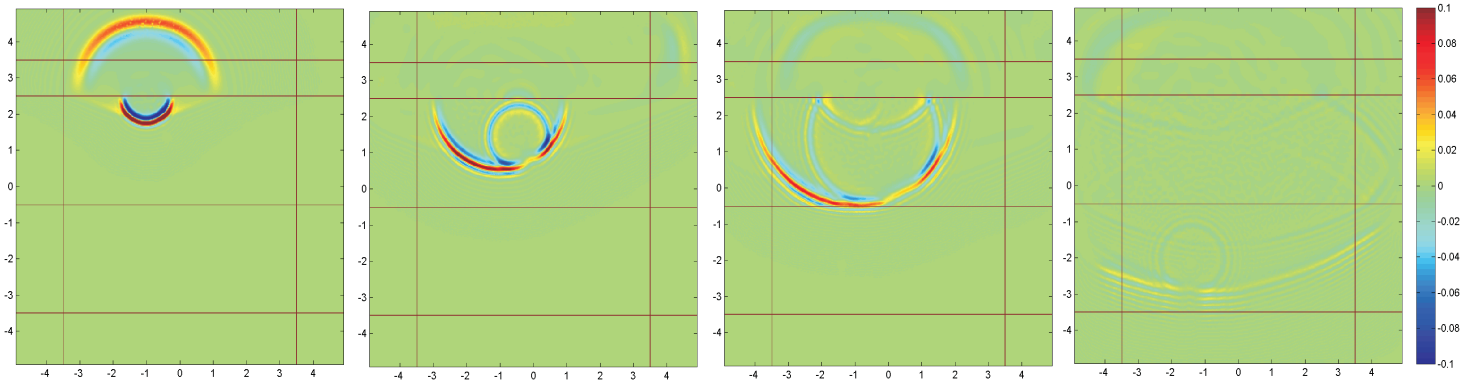

Figura 2: Fotografias mostrando amplitude do campo $\mathcal{E}_{y}$ em $t=3.38,5.92,8.46 \mathrm{~ns}$.

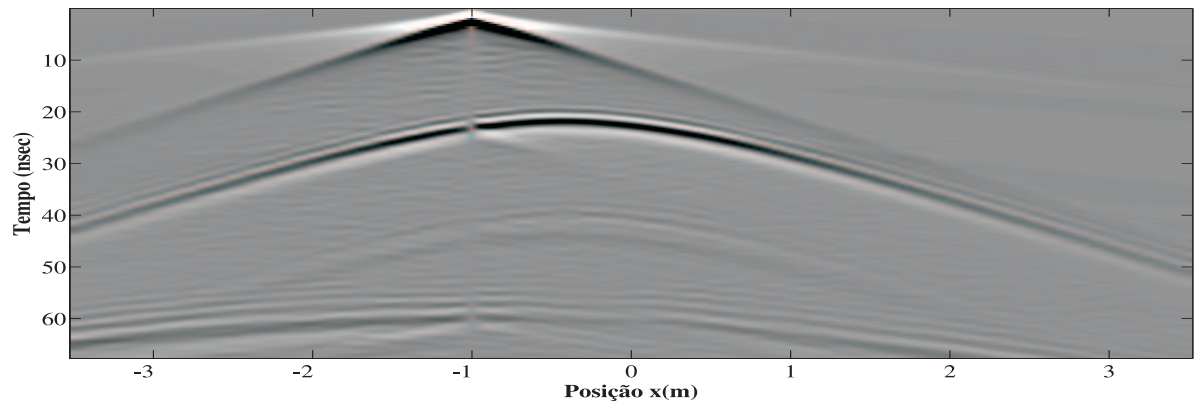

Figura 3: Componente de campo $\mathcal{E}_{y}$ observada por diversas antenas receptoras para a antena transmissora localizada em $(x, y)=(-1,2.5)$.

observa-se a eficácia da CFS-PML para o meio ar. Nota-se também a diferença de amplitude da onda nas regiões $\Omega_{1}$ e $\Omega_{2}$, devido as características elétricas dos meios.

Figura 3 mostra o comportamento da componente de campo elétrico $\mathcal{E}_{y}$ para o tempo $t$ variando de 0 a $70 \mathrm{~ns}$ e para todas as antenas receptoras localizadas na interface ar-solo $y=2.5$, a antena transmissora está localizada em $(x, y)=(-1,2.5)$. Observa-se dois tipos de eventos em Fig.3, os eventos lineares que representam a passagem direta da onda entre o ar e o solo e os eventos hiperbólicos que são os reflexos dos objetos enterrados, nos dois tipos de solo, e as reflexões causadas pelas fronteiras entre os diversos meios.

\subsection{Detecção de estruturas em concreto}

Nesta seção apresenta-se a simulação da detecção de uma estrutura em concreto. Figura 4 apresenta estrutura simulada, que é composta por um túnel com $5 \mathrm{~m}$ de largura e $3 \mathrm{~m}$ de altura construído em um solo com permissividade relativa $\epsilon_{r}=2,4$ e condutividade elétrica $\sigma=1 \times 10^{-3} \mathrm{~S} / \mathrm{m}$. Os parâmetros da função CFS-PML são $m=2,55, R=\exp (-13,37)$, $k_{\max }=4,004$ e $\alpha=0.001$, a região PML possui uma espessura de $r=2 \mathrm{~m}$, e é terminada por uma PEC.

O material do túnel é concreto com permissividade relativa $\epsilon_{r}=5,5$ e condutividade elétrica 


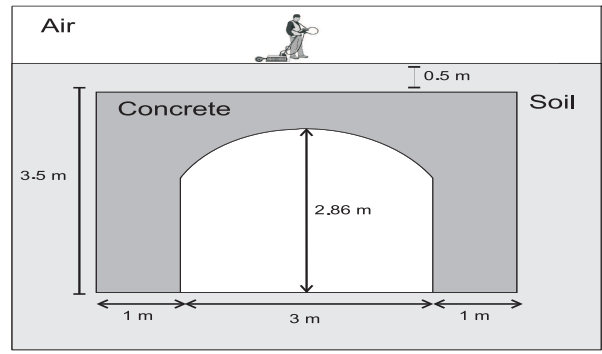

Figura 4: Esquema de deteç̧ão de estrutura em concreto por GPR, composta por túnel de concreto construído em um solo com características elétricas com perdas.

$\sigma=0 \mathrm{~S} / \mathrm{m}$. Para todos os materiais do esquema assumi-se uma permeabilidade relativa $\mu_{r}=1$. A antena transmissora é modelada como um dipolo iluminada por um pulso Blackman-Harris com frequência dominante de $500 \mathrm{MHz}$.

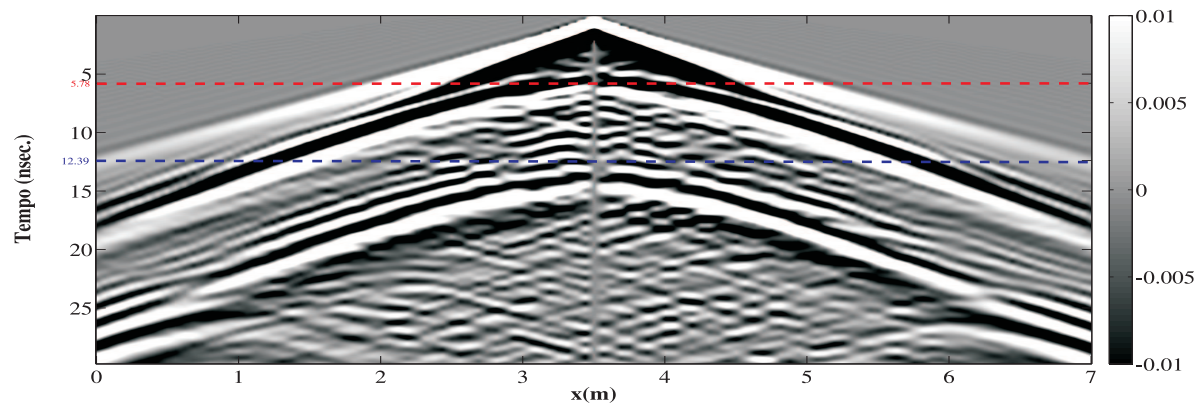

Figura 5: Campo elétrico $\mathcal{E}$ observado por antenas receptoras posicionadas na interface ar-solo para antena transmissora posicionada no centro do percurso. Linha tracejada em vermelho indica $t_{r_{1}}=5,7816 \mathrm{~ns}$ e linha tracejada em azul indica $t_{r_{2}}=12,39 \mathrm{~ns}$.

Figura 5 apresenta radargrama que corresponde a uma medição feita sobre a interface ar-solo com a presença da estrutura em concreto (túnel). Percebe-se a ocorrência de eventos hiperbólicos o que indica os efeitos de reflexãocausados pela estrutura de concreto.

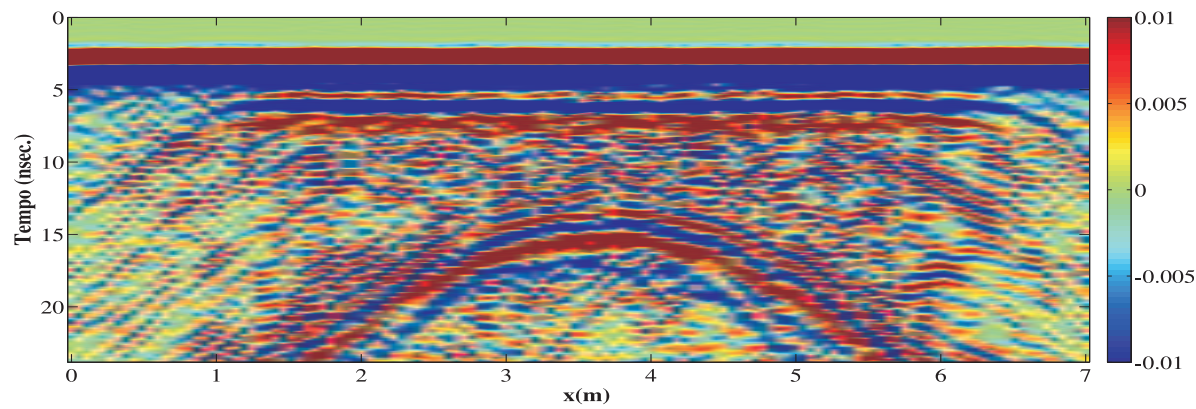

Figura 6: Radragrama obtido por antena receptora que se move simultaneamente com a antena transmissora na interface ar-solo.

Figura 6 apresenta radargrama que corresponde a uma medição feita sobre a interface arsolo para antenas transmissora e receptora se movendo simultaneamente. Novamente se tem a ocorrência de eventos hiperbólicos o que indica os efeitos de reflexão causados pela estrutura de concreto. 


\section{Conclusão}

Um algoritmo foi apresentado para se resolver as equações de Maxwell em domínios truncados pela CFS-PML. A formulação apresentada usa a teoria das formas diferenciais como base de desenvolvimento, o que torna o algoritmo extremamente simplificado independente da dimensão do problema. A discretização é baseada puramente nas matrizes de incidência e de Hodge, ou seja, através das propriedades topológicas e da aproximação via método de Galerkin. Os resultados apresentados neste artigo mostram a alta capacidade de absorção da CFS-PML.

Para se verificar a eficiência do método duas simulações de sistemas de GPR em domínios bidimensionais são apresentadas. Uma tratando o problema de levantamento de superfícies e outra a detecção de uma estrutura em concreto. Em ambos os problemas foi considerado meios com perdas.

O uso das formas diferenciais não se restringe a sistema de GPR e pode ser aplicada a qualquer problema de propagação de onda eletromagnética em domínios bi e tridimensionais. A técnica de absorção apresentada neste artigo pode ser facilmente estendida para domínios tridimensionais.

\section{Agradecimentos}

Este trabalho foi financiado em parte pela Fundação de Amparo à Pesquisa do Estado de Minas Gerais - FAPEMIG, Brasil, Conselho Nacional de Desenvolvimento Científico e Tecnológico CNPq, Brasil, Fundação de Amparo à Pesquisa do Espirito Santo - FAPES, Brasil e Coordenação de Aperfeiçoamento de Pessoal de Nível Superior - CAPES, Brasil.

\section{Referências}

[1] K. Warnick, R. Selfridge, and D. Arnold, "Teaching electromagnetic field theory using differential forms," Education, IEEE Transactions on, vol. 40, no. 1, pp. 53 -68, feb 1997.

[2] B. He and F. L. Teixeira, "Geometric finite element discretization of maxwell equations in primal and dual spaces," Phys. Lett. A, vol. 349, no. 1-4, pp. 1-14, 2006.

[3] A. S. Moura, R. R. Saldanha, E. J. Silva, M. F. Pantoja, A. C. Lisboa, and W. G. Facco, "Discretization of the cfs-pml for computational electromagnetics using discrete differential forms," Microwave and Optical Technology Letters, vol. 55, no. 2, pp. 351-357, 2013. [Online]. Available: http://dx.doi.org/10.1002/mop.27298

[4] A. Bossavit, "Whitney forms: a class of finite elements for three-dimensional computations in electromagnetism," Physical Science, Measurement and Instrumentation, Management and Education - Reviews, IEE Proceedings A, vol. 135, no. 8, pp. 493 -500, nov 1988.

[5] — Discretization of Electromagnetic Problems: The Generalized Finite Differences Approach, ser. in W.H.A. Schilders, E.J.W. Ter Maten (Eds): Numerical Methods in Electromagnetism (Handbook of Numerical Analysis, Vol. 13). Elsevier (Amsterdam), 2005.

[6] —- Computational Electromagnetism: variational formulation, complementarity, edge elements. San Diego: Academic Press, 1994.

[7] A. S. Moura, R. R. Saldanha, E. J. Silva, A. C. Lisboa, W. G. Facco, and N. Z. Lima, "A recursive sparsification of the inverse hodge matrix," Magnetics, IEEE Transactions on, vol. 48, no. 2, pp. $611-614$, feb. 2012. 